\title{
¿Lobo o Lóbulo? Una Explicación desde el Origen Etimológico: Propuesta para Terminologia Anatomica
}

\author{
Lobe or Lobule? An Explanation from the \\ Etymological Origin: Proposal for Terminologia Anatomica \\ Rodrigo Lizama Pérez ${ }^{1,2}$; Nicolás Vidal-Seguel ${ }^{2,3,4}$; Marcela Pumeyrau Solar ${ }^{3,5}$; \\ Rodrigo Muñoz Cofrée; Daniel Conei ${ }^{6,7}$; Christopher Nicholson ${ }^{8}$ \& Bélgica Vásquez ${ }^{9}$
}

\begin{abstract}
LIZAMA, P. R.; VIDAL-SEGUEL, N.; PUMEYRAU, S. M.; MUÑOZ, C. R.; CONEI, D.; NICHOLSON, C. \& VÁSQUEZ, B. ¿Lobo o lóbulo? Una explicación desde el origen etimológico: Propuesta para Terminologia Anatomica. Int. J. Morphol., 38(4):1047-1052, 2020.

RESUMEN: Los antiguos anatomistas renacentistas sentaron las bases del conocimiento actual en esta disciplina, sin embargo, no todos eran buenos lingüistas. Esto, vinculado a los errores en la traducción, ha dado lugar para la discusión sobre el correcto uso de algunos términos en anatomía, como es el caso de la controversial utilización de los términos lobo y lóbulo. El objetivo de este estudio fue analizar los términos lobus y lobulus y proponer un concepto correcto para la denominación de las estructuras anatómicas según corresponda, siguiendo las recomendaciones de la Federative International Programme for Anatomical Terminology (FIPAT). Se realizó una búsqueda de los términos lobo y lóbulo con sus derivados en Terminologia Anatomica, cada uno de estos fue analizado desde un punto de vista etimológico y semántico según el Diccionario Médico-Biológico, Histórico y Etimológico de la Universidad de Salamanca. Los términos derivados de lobo y lóbulo se encuentran en variados sistemas del cuerpo humano, esto lo clasifica como un término de gran uso. En conclusión, el término lobo del latín lobus, proviene del griego que significa, estructura redondeada y saliente de un órgano. Posteriormente los romanos latinizaron esta palabra con el propósito de recaracterizar el término hacia el lenguaje médico. Por tanto, según el punto de la normativa FITAP, que indica al latín como idioma oficial, los términos correctos a utilizar son lobus y lobulus.
\end{abstract}

PALABRAS CLAVE: Lobo; Lóbulo; Terminologia Anatomica.

\section{INTRODUCCIÓN}

Al momento de denominar a una estructura anatómica, lo indicado es seguir las recomendaciones vigentes y validadas a nivel internacional, redactadas en la actualidad por Terminologia Anatomica (Vásquez \& del Sol, 2015; Pacheco \& Lizama, 2019). A pesar de los constantes aportes y publicaciones para mejorar y establecer criterios en la denominación de los términos, aún existen discordancias para nombrar algunas estructuras entre los distintos autores (Fuentes et al., 2016). Esto, si lo llevamos a un ambiente universitario, sin duda dificulta el aprendizaje por parte de los estudiantes (Roa et al., 2016).

Estas dificultades para denominar estructuras anatómicas, se observan de manera frecuente al momento de uti- lizar el término lobo o lóbulo (Pró, 2012; García-Porrero \& Hurlé, 2005). En este contexto, la sinonimia provocada por la transliteración de un término griego al latín es frecuente. Además, ésta se puede asociar a la evolución permanente de una lengua, factores que en su conjunto generan estas pequeñas variaciones, las cuales se mantienen en el tiempo (Navarro, 1997).

En su tratado Testut \& Latarjet (1969), en relación a los lóbulos del sistema respiratorio indican que: «las cisuras se extienden en profundidad hasta la proximidad del hilio. Dividen de este modo cada pulmón en segmentos más pequeños llamados lóbulos». Más adelante, García-Porrero \& Hurlé señalaron que: «las cisuras separan porciones del

\footnotetext{
${ }^{1}$ Departamento de Ciencias Morfológicas, Facultad de Medicina y Ciencia, Universidad San Sebastián, General Lagos 1163, Valdivia 509000, Chile.

${ }^{2}$ Programa de Magíster en Ciencias mención Morfología, Universidad de La Frontera, Temuco, Chile.

${ }^{3}$ Escuela de Enfermería, Facultad de Salud, Universidad Santo Tomás, Chile.

${ }^{4}$ Becario CONICYT-PCHA/MAGISTER NACIONAL/ 2017-22170070.

${ }^{5}$ Programa de Magíster en Lingüística Aplicada, Facultad de Humanidades y Arte, Universidad de Concepción, Concepción, Chile.

${ }^{6}$ Programa de Doctorado en Ciencias Morfológicas, Universidad de La Frontera, Temuco, Chile.

${ }^{7}$ Departamento de Ciencias de la Salud, Universidad de Aysén, Coyhaique, Chile.

${ }^{8}$ Núcleo de Idiomas, Facultad de Estudios Interdisciplinarios, Universidad Mayor, Temuco, Chile.

${ }^{9}$ Universidad de Tarapacá, Chile.
} 
parénquima pulmonar de forma parcial, visibles en la superficie y profundizan al interior del órgano para dividirlos en lóbulos». No queda duda que el término lóbulo, en anatomía, se asocia a la porción de un órgano delimitado por fisuras, surcos o tejido conectivo (Strasberg, 2005). Sin embargo, en la actualidad la discusión instaurada en el ámbito de la anatomía, es si efectivamente el término correcto a utilizar es lobo o lóbulo.

Los términos lobo y lóbulo son definiciones idénticas y podrían eventualmente ser utilizadas para definir la misma estructura en el cuerpo humano (Real Academia Nacional de Medicina de España, 2011). Sin embargo, la Terminologia Anatomica los interpreta como dos conceptos distintos, en consecuencia, lobus y lobulus representarían estructuras diferentes en el cuerpo humano.

En este contexto, el objetivo de este estudio fue analizar los términos lobus y lobulus, y así proponer un concepto correcto para la denominación de las estructuras anatómicas según corresponda, siguiendo las recomendaciones de la Federative International Programme for Anatomical Terminology (FIPAT).

\section{MATERIAL Y MÉTODO}

Realizamos una búsqueda de los términos que contenían los conceptos lobo y lóbulo con sus derivados en Terminologia Anatomica (FCAT, 1998) y en la segunda edición de Terminologia Anatomica de la FIPAT, un Programa de la Federación Internacional de Asociaciones de Anatomistas (IFAA) de 2019, cuya publicación se encuentra pendiente y debería haber sido aprobada en el Congreso Internacional de la IFAA realizado recientemente en Londres.
Es necesario señalar que la segunda edición de Terminologia Anatomica se desarrolló a partir de la primera edición. Sin embargo, reimpresiones de la primera edición se emitieron por error en 2011, como la «seguna edición» (FIPAT, 2019).

Cada uno de los términos lobo y lóbulo fue analizado desde un punto de vista etimológico y semántico según las directrices propuestas por el Diccionario Médico-Biológico, Histórico y Etimológico de la Universidad de Salamanca (Cortés \& Ureña, 2019). Posteriormente, se analizó el Diccionario de Términos Médicos (Real Academia Nacional de Medicina) y se buscaron las definiciones de cada uno de los términos relacionados. Esto mismo se realizó con el Diccionario de la Lengua Española (RAE, 2014). Para finalizar, los conceptos se tabularon en una tabla y se realizaron las propuestas correspondientes.

\section{RESULTADOS}

La etiología del término lobo del latín lobu(s), proviene del griego [خoßós], cuya traducción al español es lobo. En cuanto al término lóbulo, este proviene del griego $[\lambda \circ \beta O ́ \varsigma]+-u l u(m)$, del latín "pequeño" (Cortés \& Ureña).

En el Diccionario de Términos Médicos (Real Academia Nacional de Medicina), 62 términos se asocian a la raíz lobo o lóbulo. 52 de ellos son sustantivos masculinos, 5 sustantivos femeninos y 5 adjetivos (Tabla I). En todos, se aclara que la raíz ' $l o b u(s)$-' es de origen griego y se traduce como lóbulo al español. En el caso del término lóbulo y sus derivados, este se encuentra compuesto de la raíz lob $(u)+$ $u l u(m)$. Este término cuenta con dos definiciones: i) «Porción de un órgano delimitada por cisuras, surcos o tabiques

Tabla I. Términos con raíz lobo o lóbulo en Diccionario de Términos Médicos de la Real Academina Nacional de Medicina de España.

\begin{tabular}{|c|c|c|}
\hline Sustantivo masculino & Sustantivo femenino & Adjetivo \\
\hline $\begin{array}{l}\text { Lobulillo, Lobulillo clásico, Lobulillo cuadrado del cerebro, Lobulillo cuadrangular, } \\
\text { Lobulillo cuadrangular del cerebelo, Lobulillo cuadrilátero del cerebelo, Lobulillo de } \\
\text { la glándula mamaria, Lobulillo hepático, Lobulillo hepático clásico, Lobulillo HIX, } \\
\text { Lobulillo mamario, Lobulillo portal, Lobulillo pulmonar, Lobulillo pulmonar } \\
\text { primario, Lobulillo pulmonar secundario, Lobulillo respiratorio, Lobulillo VI del } \\
\text { vermis. } \\
\text { Lóbulo, Lóbulo anterior del cerebelo, Lóbulo caudado, Lóbulo caudal del cerebelo, } \\
\text { Lóbulo cerebral, Lóbulo craneal del cerebelo, Lóbulo cuadrado, Lóbulo cuadrado del } \\
\text { hígado, Lóbulo cuadrangular del cerebelo, Lóbulo cuneiforme, Lóbulo falciforme, } \\
\text { Lóbulo floculonodular, Lóbulo frontal, Lóbulo de la glándula mamaria Lóbulo de la } \\
\text { glándula tiroidea, Lóbulo hepático, Lóbulo del hígado } \\
\text { Lóbulo de la ínsula, Lóbulo límbico, Lóbulo mamario, Lóbulo medio de la próstata, } \\
\text { Lóbulo occipital, Lóbulo de la oreja, Lóbulo parietal, Lóbulo posterior del cerebelo, } \\
\text { Lóbulo posterior de la hipófisis, Lóbulo prefrontal, Lóbulo prostático, Lóbulo } \\
\text { prostático medio, Lóbulo pulmonar, Lóbulo renal, Lóbulo rostral del cerebelo, } \\
\text { Lóbulo de Spiegel, Lóbulo temporal, Lóbulo tiroideo. }\end{array}$ & $\begin{array}{l}\text { Lobectomía } \\
\text { Lobotomía } \\
\text { Lobotomía frontral } \\
\text { Lobotomía prefrontal } \\
\text { Lobulectomía }\end{array}$ & $\begin{array}{l}\text { Lobar } \\
\text { Lobulado, -da } \\
\text { Lobular } \\
\text { Lobuliforme } \\
\text { Lobulillar }\end{array}$ \\
\hline
\end{tabular}


Tabla II. Uso de los términos lobus y lobulus en Terminologia Anatomica, 1998.

$\begin{array}{cc}\text { Sistemas donde se utiliza el término lobus } \\ \text { Código } & \text { Sistema } \\ \text { A05.1.00.000 } & \text { Systema digestorium } \\ \text { A06.0.00.000 } & \text { Systema respiratorium } \\ \text { A09.0.00.000 } & \text { Systemata genitalia } \\ \text { A11.0.00.000 } & \text { Glandulae endocrinae } \\ \text { A13.0.00.000 } & \text { Systema lymphoideum } \\ \text { A14.0.00.000 } & \text { Systema nervosum } \\ \text { Sistemas donde se utiliza el término lobulus } \\ \text { Código } & \text { Sistema } \\ \text { A06.0.00.000 } & \text { Systema respiratorium } \\ \text { A09.0.00.000 } & \text { Systemata genitalia } \\ \text { A14.0.00.000 } & \text { Systema nervosum } \\ \text { A15.1.00.001 } & \text { Organa sensuum } \\ \text { Sistemas donde se utiliza el término lobi } \\ \text { Código } & \text { Sistema } \\ \text { A08.0.00.000 } & \text { Systema urinarium } \\ \text { A09.0.00.000 } & \text { Systemata genitalia } \\ \text { A12.0.00.000 } & \text { Systema cardiovasculare } \\ \text { A14.0.00.000 } & \text { Systema nervosum } \\ \text { A05.1.00.000 } & \text { Systema digestorium } \\ \text { A16.0.00.001 } & \text { Integumentum commune } \\ \text { Sistemas donde se utiliza el término lobuli } \\ \text { Código } & \text { Sistema } \\ \text { A05.1.00.000 } & \text { Systema digestorium } \\ \text { A09.0.00.000 } & \text { Systemata genitalia } \\ \text { A11.0.00.000 } & \text { Slandulae endocrinae } \\ \text { A13.0.00.000 } & \text { Systema nervosum } \\ \text { A14.0.00.000 } & \text { Integumentum commune } \\ \text { A16.0.00.001 } & \\ & \end{array}$

Tabla III. Uso de los términos lobus y lobulus en Terminologia Anatomica, 2019.

\begin{tabular}{|c|c|}
\hline \multicolumn{2}{|c|}{ Sistemas donde se utiliza el término lobus } \\
\hline Código & Sistema \\
\hline 2773 & Systema digestorium \\
\hline 3133 & Systema respiratorium \\
\hline 3357 & Systema urinarium \\
\hline 3574 & Systema genitale masculinum \\
\hline $3851(3852)$ & Systema integrantia (Glandulae endocrinae) \\
\hline 3891 & Systema cardiovasculare \\
\hline 5149 & Systema lymphoideum \\
\hline 5363 & Systema nervosum \\
\hline \multicolumn{2}{|c|}{ Sistemas donde se utiliza el término lobulus } \\
\hline Código & Sistema \\
\hline 3133 & Systema respiratorium \\
\hline 3574 & Systema genitale masculinum \\
\hline $3851(3852)$ & Systema integrantia (Glandulae endocrinae) \\
\hline 3891 & Systema cardiovasculare \\
\hline 5363 & Systema nervosum \\
\hline \multicolumn{2}{|c|}{ Sistemas donde se utiliza el término $l o b i$} \\
\hline Código & Sistema \\
\hline 2773 & Systema digestorium \\
\hline 3574 & Systema genitale masculinum \\
\hline 3891 & Systema cardiovasculare \\
\hline 5363 & Systema nervosum \\
\hline 7040 & Integumentum commune \\
\hline \multicolumn{2}{|c|}{ Sistemas donde se utiliza el término lobuli } \\
\hline Código & Sistema \\
\hline 2773 & Systema digestorium \\
\hline 3574 & Systema genitale masculinum \\
\hline $3851(3852)$ & Systema integrantia (Glandulae endocrinae) \\
\hline 5149 & Systema lymphoideum \\
\hline 5363 & Systema nervosum \\
\hline 7040 & Integumentum commune \\
\hline
\end{tabular}

de tejido conjuntivo, como los lóbulos hepáticos, cerebrales o pulmonares», y ii) «Porción redondea, cilíndrica o cónica, habitualmente carnosa, que sobresale de un órgano, como el lóbulo de la oreja».

En Terminologia Anatomica los términos derivados de lobo y lóbulo, en los distintos sistemas, se encuentran en las Tablas II y III. También se encuentran los derivados lobi y lobuli, los que tendrían su raíz en las palabras lobo y lóbulo, respectivamente.

Desde el punto de vista histórico, los registros indican que el término lobo fue utilizado por primera vez en el siglo VIII a.C., por Homero en su obra La Ilíada, para referirse al lóbulo de la oreja por su forma redondeada (LSJ, 2019). Posteriormente, este concepto se fue utilizando en otros órganos para finalmente ser definido en la actualidad como "una estructura redondeada y saliente de un órgano" (Strasberg; RAE).

\section{DISCUSIÓN}

Antes de analizar los términos lobus y lobulos, se hace necesario señalar que la actual Terminologia Anatomica se divide en 16 capítulos, agrupados en cinco partes. Lo interesante, y que ha cambiado, es que el código de identificación de cuatro partes de la primera edición, que era utilizado de manera inconsistente según la FIPAT, ha sido reemplazado por un sistema estándar de números de identificación únicos. Los términos oficiales de la FIPAT siguen estando en latín, aunque se proporcionan los términos equivalentes en inglés, que son de mucha utilidad al escribir artículos en ese idioma. Sin embargo, hay que recordar que la Nomina Anatomica de Basilea, Jena y París y, en sus ediciones posteriores, al igual que Terminologia Anatomica, han señalado que sólo desde el latín, como base, se pueden crear listas de términos equivalentes en otros idiomas.

Esta recomendación se ha seguido en numerosos países del mundo y en sus respectivos idiomas, así, por ejemplo, en inglés lobus será «lobe» y lobulus será «lobule» y en portugués lobus será «lobo»y lobulus será «lóbulo». No obstante lo anterior, en otros países, incluido los de habla hispana, no han seguido del todo las recomendaciones de la FIPAT. Particularmente para los términos lobus y lobulus se hace complejo trasladarlos al español, ya que el término lobus no está incluido en el Diccionario de Términos Médicos (Real Academia Nacional de Medicina).

Bajo este contexto, ¿qué sucede con el idioma español? Parece que, en algunas oportunidades, los profesionales médicos y del área de la salud se resisten al cambio, en especial en el uso adecuado de términos anatómicos. Basta señalar algunos ejemplos, el término lobar, incluido en el Diccionario de Términos Médicos (Real Academia Nacional de Medicina), tiene una observación: «puede suscitar rechazo, por considerarse anglolatinismo innece- 
sario, pero es sumamente frecuente, sobre todo en la expresión neumonía lobar». También están incorporados los términos médicos de lobectomía, lobotomía y otros, pero otra vez con una observación, señalando que es una extirpación de un lóbulo o de una glándula. Nuevamente se indica: «puede suscitar rechazo por considerarse incorrectamente formado; la forma lobulectomía es más correcta, pero de uso minoritario».

Como se puede observar en los párrafos anteriores, el problema no radica en la Terminologia Anatomica, que son los términos oficiales de la FIPAT en latín, los cuales tienen una correcta equivalencia en inglés, el idioma científico. Sino más bien, el problema se suscita porque en el Diccionarios de la Lengua Española (RAE) no se hace mención a la acepción anatómica del término lobo.

Un ejemplo de las divergencias ocasionadas entre lobus y lobulus es el término Lobulus centralis vermis, en el cual el comité de la FIPAT consideró que lobulus era un nombre más apropiado para esta pequeña estructura que lobus, término que es utilizado en la Nomina Anatomica de París, 1955. La denominación Lobulus centralis vermis también fue considerada en Nomina Anatomica de 1961 y en la presente Terminologia Anatomica de 2019.

Otro aspecto a considerar es el caso del término lóbulo del latín lobulum, cuyo sufijo -ulum se usa para identificar diminutivo. Situación que ha impedido analizar convenientemente su capacidad categorizadora, la cual, es la base del lenguaje técnico en anatomía (López, 2005; Criado-de Diego \& Andión-Herreo, 2016). Junto con lo anterior, es importante aclara, que los términos lobi y lobuli son la declinación nominativa plural de las palabras lobus y lobulum, respectivamente.

En la lengua latina, el diminutivo se utiliza para el lenguaje especializado asociado al léxico agrícola y posteriormente al médico, con el propósito de recaracterizar y lograr mayor expresividad de una palabra sin que ésta pierda su significado (Ferreira, 2010). Esta situación no es menor, considerando que algunos textos en la actualidad utilizan el término lobulillo (Tabla I) para expresar diminutivo. Este lo definen como: «unidad estructural del parénquima de un órgano, de carácter prismático o piramidal, delimitada completamente o parcialmente por tabiques de tejido conjuntivo. Varios lobulillos constituyen un lóbulo». En este punto, la FIPAT es clara en cuanto a que en Terminologia Anatomica el idioma oficial es el latín, por tanto, el término lobulillo debería ser reemplazado por lóbulo. Así, varios lóbulos conforman un lobo.

En el mundo de la medicina, el Diccionario de Términos Médicos reúne una serie de palabras con un amplio uso en las áreas del quehacer médico. Aquí, la definición dada para lóbulo es, «órgano determinado restringido por cisuras, surcos o tabiques de tejido conectivo», dando ejemplos como los lóbulos cerebrales, hepáticos o pulmonares. Además, para las porciones más pequeñas de un lóbulo que conforman su parénquima y que a su vez se encuentran de-

Tabla IV. Definiciones del Diccionario de la Lengua Española (RAE).

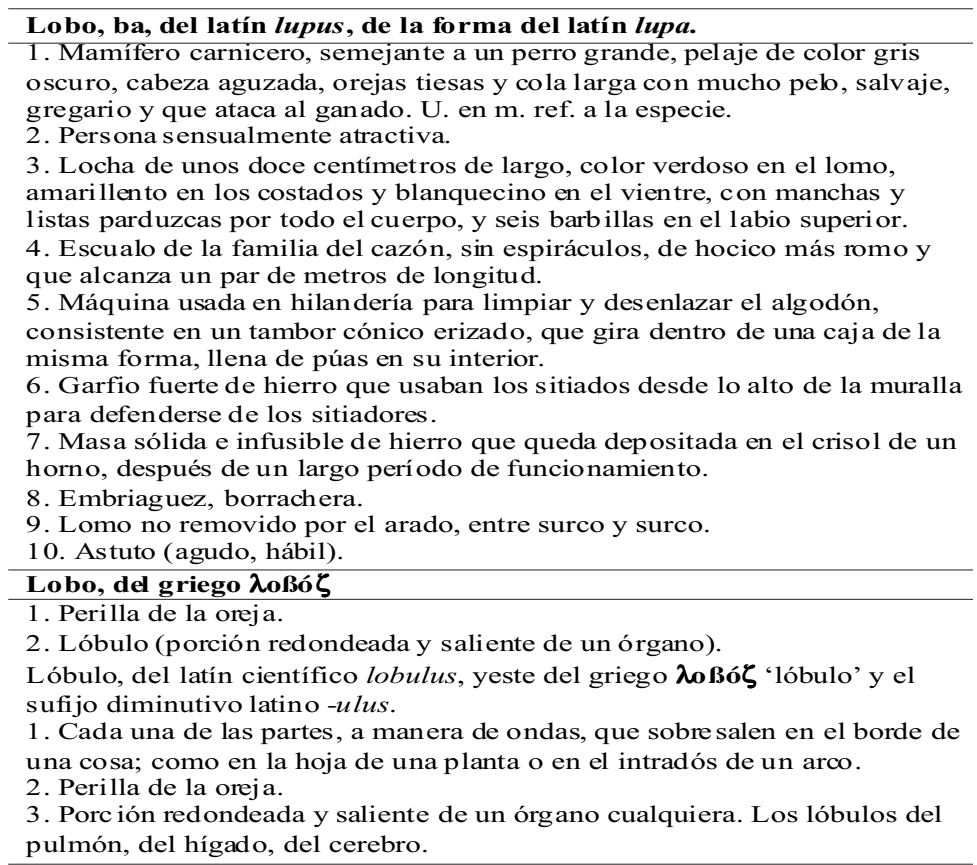


limitados por tejido conectivo, se definen como lobulillos. También especifica que varios de ellos forman un lóbulo (Real Academia Nacional de Medicina). Esto se contrapone con lo sugerido por la FIPAT, la cual ya cuenta con una serie de normas para estandarizar los términos a utilizar en anatomía. En este contexto, la sugerencia es aunar criterios y consultar a las instituciones especializadas, para disminuir al máximo la sinonimia.

Otra de las fuentes principales de información a consultar, al momento de despejar dudas de un significado, es el Diccionario de la Lengua Española. Este indica, dentro de sus muchas definiciones, que lobo es «un mamífero carnicero, semejante a un perro grande, pelaje de color gris oscuro, cabeza aguzada, orejas tiesas y cola larga con mucho pelo, salvaje, gregario y que ataca al ganado». Además, hace una distinción en su significado anatómico en donde lo describe como «la perilla de la oreja» y lóbulo como «la porción redondeada y saliente de un órgano» (RAE). $\mathrm{Mu}-$ chos términos, tanto latinos como griegos, que se han incorporado a distintos idiomas en diferentes épocas de su historia, han adquirido significados diferentes en su equivalente castellano (Tabla IV). De este modo, la polisemia es un fenómeno frecuente en el léxico latino ( López; García, 2010; Sánchez, 2015). En algunos casos no resulta problemático, ya que los términos poseen un significado general y otro especializado, los cuales son en contexto distintos. En este punto es importante destacar que, en el caso del término lobo, más que un doble significado, es una modificación fonética vinculada a una connotación popular, situación que ha perdurado en el tiempo.

En este mismo caso, existió una modificación fonética, que pudo haber generado una confusión en el origen de la palabra lobo. Entre los idiomas derivados del latín, el portugués y español tienen una fuerte proximidad gracias a factores históricos (Sánchez). Si bien, ambos recibieron influencias similares de los dialectos escandinavos, germánico y árabe, producto de invasiones militares y largos períodos de ocupación, su mayor ascendencia es el latín. Con la conquista romana de los pueblos celtíberos y la incorporación de la península al imperio, se produce un proceso de aculturación, donde el latín se impone como lenguaje hablado y escrito. Durante los casi ocho siglos de dominio romano, Hispania, situada en el final del imperio, tuvo poco contacto con las otras colonias romanas, situación por la cual no conoció las innovaciones posteriores de Roma (García). Asociado a este proceso, el término lobo evolucionó en uno de sus fonemas. Específicamente, los fonemas oclusivos bilabiales y linguodentales sordos p/e/t de origen latino, migraron a fonemas de sonidos oclusivos bilabiales y linguales b/e/d en el medio de las palabras (Custódio \& Pastafiglia, 2006). Por este mecanismo, el término lobo, pro- viene del portugués lobo, el que a su vez deriva del término latín lupus, «mamífero carnicero, semejante a un perro grande, pelaje de color gris oscuro, cabeza aguzada, orejas tiesas y cola larga con mucho pelo, salvaje, gregario y que ataca al ganado». Además de esto, lupus, en la antigüedad era usado para varias enfermedades que causan ulceraciones de la piel, debido a que «devora» la parte afectada, se generó el vínculo con el animal y forma de comer.

En relación a su connotación popular en el tiempo, en la antigua Roma, lupa «loba» (conservada en español, loba), era la denominación entregada a una mujer sexualmente voraz. Este vínculo, lobo-prostituta, persistió hasta el siglo XIII y se expandió por el vasto imperio romano. Años después a principios del siglo XVIII, en los países escandinavos, se utilizaba al lobo como símbolo de lujuria (Online Etymology Dictionary, 2019). Por tanto, una teoría al respecto sería que esta evolución del término lupus a lobo y, lo que representaba en tiempos antiguos, le confirió esta relación con el animal, alejándolo erróneamente del significado anatómico, el cual, proviene del latín lobus.

En conclusión, el término lobo del latín lobus, proviene del griego $[\lambda \circ \beta O ́ \varsigma]$ que significa, estructura redondeada y saliente de un órgano. Posteriormente los romanos latinizaron esta palabra con el propósito de recaracterizar el término hacia el lenguaje médico. Por tanto, según el punto de la normativa FIPAT, que indica al latín como idioma oficial, los término correctos a utilizar son lobo del latín lobus y lóbulo del latín lobulus, para una subunidad de un lobo. A su vez, se recomienda que este tipo de conclusiones tengan un impacto no sólo en la comunidad científica, sino también en el ámbito de la lingüística como en lo que recomienda y orienta la Real Academia Española y muy especialmente en Terminologia Anatomica.

LIZAMA, P. R.; VIDAL-SEGUEL, N.; PUMEYRAU, S. M.; MUÑOZ, C. R.; CONEI, D.; NICHOLSON, C. \& VÁSQUEZ,

B. Lobe or lobule? An explanation from the etymological origin: Proposal for Terminologia Anatomica. Int. J. Morphol., 38(4):10471052, 2020.

SUMMARY: During the Renaissance anatomists laid the foundations for present day knowledge in this discipline. Not all anatomists however, were good linguists, which led to errors in translation and prompted discussions in reference to the correct use of certain anatomical terms. The controversial use of the terms lobe and lobule being one of these discrepancies. The objective of this study was to analyze the terms lobus and lobulus and propose a correct concept for the naming of anatomical structures following Federative International Programme for Anatomical Terminology (FIPAT) recommendations. A search was carried out for the terms lobe and lobule with their derivatives in Anatomical Terminology. 
Each term was analyzed from an etymological and semantic point of view according to the Medical-Biological, Historical and Etymological Dictionary, University of Salamanca. Terms derived from lobe and lobule can be found in a number of systems in the human body, characterizing this term as one of frequent use. In conclusion, the term lobe from the Latin lobus, originates from the Greek language, meaning the rounded structure and protrusion of an organ. Subsequently, Romans Latinized the term in order to reclassify it into medical language. Consequently, and in accordance to FITAP regulation, with Latin as the official language, the correct terms to use are lobus and lobulus.

KEY WORDS: Lobe; Lobule; Anatomical Terminology.

\section{REFERENCIAS BIBLIOGRÁFICAS}

Criado-de Diego, C. \& Andión-Herrero, M. Variación y variedad del diminutivo en español a través de dos corpus originales. Apuntes para su enseñanza como lengua extranjera. Rev. Electron. Lingüís. Apl., 15(1):87-108, 2016.

Comité International de la Nomenclature Anatomique. Nomina Anatomica. París, 1955.

Cortés, F. \& Ureña, J. Diccionario médico-biológico, histórico y etimológico. Salamanca, Ediciones Universidad de Salamanca, 2019. https:// dicciomed.usal.es/palabra/lobo

Custódio, R. M. \& Pastafiglia, M. Aplicação das regras gerativas nas mudanças fonéticas do espanhol e do português na evolução do latim. Rev. Inic. Cient., 4(1):81-9, 2006.

Federative Committee on Anatomical Terminology (FCAT). Terminologia Anatomica: International Anatomical Terminology. Stuttgart, Georg Thieme Verlag, 1998.

FIPAT. Terminologia Anatomica. $2^{\text {nd }}$ ed. FIPAT.library.dal.ca. Federative International Programme for Anatomical Terminology, 2019.

Ferreira M. A. I. Medicina y filología de estudios léxico médico latino en la edad media. Fédération Internationale des Instituts d'Études Médiévales, Porto, Portugal, 2010.

Fuentes, R.; Ottone, N. E.; Bucchi, C. \& Cantín, M. Analysis of terms used in the literature to refer to temporomandibular joint capsule and joint ligaments. Int. J. Morphol., 34 (1):342-50, 2016.

García, C. La formación de la terminología anatómica en español (14931604). Tesis doctoral. Salamanca, Universidad de Salamanca, 2010.

García-Porrero, J. \& Hurlé, J. Anatomía Humana. Madrid, McGraw-Hill, 2005.

López, G. M. R. El uso del diminutivo en el lenguaje técnico latino. Relat., 5, 75-96, 2005.

LSJ. The Online Liddell-Scott-Jones Greek-English Lexicon. 2019. http:// stephanus.tlg.uci.edu/lsj/\#eid=65772.

Navarro, F. A. Traducción y Lenguaje en Medicina. Barcelona, España. Ediciones Doyma, 1997.

Online Etymology Dictionary. https://www.etymonline.com/search?q=lobo. 11 de octubre de 2019.

Pacheco, J. P. \& Lizama, P. R. Tubérculo de Gerdy. Una estructura olvidada en la Terminología Anatómica Internacional. Int. J.Morphol., 37(4):13059, 2019.

Pró, E. A. Anatomía Clínica. $2^{\mathrm{a}}$ ed. Buenos Aires, Médica Panamericana, 2012. Real Academia Española (RAE). Diccionario de la lengua española. 23. ${ }^{\mathrm{a}} \mathrm{ed}$., Madrid, Espasa, 2014. Disponible en: https://dle.rae.es/lobo?m=form

Real Academia Nacional de Medicina. Diccionario de Términos Médicos. Panamericana, Madrid, 2011.

Roa, I.; Vásquez, B. \& Contreras, M. Persistencia de Epónimos en Terminologia Histologica. Int. J. Morphol., 34(4):1245-52, 2016.

Sánchez, L.A. La formación del registro metalingüístico en latín tardorrepublicano (siglo I a.C.). El testimonio del De Lingua Latina de Varrón. Rev. Argent. Historiogr. Lingüíst., VII, 1, 69-82, 2015.
Strasberg, S. M. Nomenclature of hepatic anatomy and resections: a review of the Brisbane 2000 system. J. Hepatobiliary Pancreat. Surg., 12(5):351$5,2005$.

Vásquez, B \& del Sol, M. Terminologia Anatomica y Terminologia Histologica: Un Lugar de Encuentro entre los Morfólogos. Int. J. Morphol., 33(4):158590, 2015.

Testut, L. \& Latarjet, A. Tratado de Anatomía Humana. 9ª ed. Barcelona Salvat, 1969.

\author{
Dirección de correspondencia: \\ Rodrigo Lizama Pérez \\ Departamento de Ciencias Morfológicas \\ Facultad de Medicina y Ciencia \\ Universidad San Sebastián \\ General Lagos 1163 \\ Valdivia 5090000 \\ CHILE
}

Email: rodrigo.lizama@uss.cl

Recibido : 11-10-2019

Aceptado: 08-03-2020 